NASA-CR-201038

Procedure for Adapting Direct Simulation

Monte Carlo Meshes

M S. Woronowicz

Vignon, Inc., Hampton, Virginia

and

R. G. Wilmoth, A.B. Carlson, and D. F. G. Rault

NASA Langley Research Center, Hampton, Virginia

DAIAA:

Reprinted from Rarefied Gas Dynamics: Theory and Simulations, edited by Bernie D. Shizgal and David P. Weaver, Vol. 159 of Progress in Astronautics and Aeronautics, AIAA, Washington, DC, ISBN 1-56347-08()-2. 



\title{
Procedure for Adapting Direct Simulation Monte Carlo Meshes
}

\author{
Michael S. Woronowicz* \\ Vigyan. Inc.. Hampton, Virginia 23600 \\ and \\ Richard G. Wilmoth $\uparrow$ Ann B. Carlson, $\uparrow$ and Didier F. G. Rault $\dagger$ \\ NASA Langley Research Center, Hampton, Virginia 23681
}

\begin{abstract}
A technicue is presented for adapting computational meshes used in the ( $i 2$ version of the direct simulation Monte (arlo method. The physical ideas underlying the technique are discussed, and adaptation formulas are developed for use on solutions generated from an initial mesh. The effect of statistical scatter on adaptation is addressed, and results demonstrate the alility of this technigue to achieve more accurate results without inc reasing necessary computational resources.
\end{abstract}

\section{Introduction}

In recent years, statistical particle methods, such as the direct simulation Monte (arlo method (DSMC), have become a popular approach for simulating high-speed, rarefied gas flows. ${ }^{1-4}$ However, their widespread use is often hampered by heavy demands on computational resources, such as memory and CPU time. In addition, investigators must often use their initial results as a guideline for obtaining more accurate answers by modifying initial spatial or temporal resolution. This iterative process increases the amount of user time, and often the amount of computational resources, necessary to compute an accurate solution.

The purpose of this investigation is to examine algorithms for automatically adapting grids to initial DSM(: solutions using a number of seemingly conflicting criteria for accuracy. With such procedures, we expect to roduce the memory, (PU time, and user time necessary to achieve satisfactory le'vels of accuracy. The algorithms examined here are compatible with the (i2 version of the IDSM : method.

Copyright $(1) 1992$ by the American Institute of Aeronautics and Astronatitios, Inc. All rights reserved.

* Research Scientist.

† Researcl Scientist, Aerothermodynanics Branch. 


\section{Discussion}

From experience with DSMC simulations, users have developed accuracy criteria with respect to grid resolution. While it is unnecessary to obey these rules everywhere at all times, following them provides some confidence that the final results are as accurate as the method permits."

One rule maintains that in viscous layers, shock waves, and shear layers, where macroscopic properties undergo strong changes, cell spacing along these gradients should not exced one local mean free path length, $\lambda$. This ensures that properties transported across these gradients through interparticle collisions are captured properly

Another rule requires that near surfaces, cell spacing normal to the body should also be shorter than $\lambda$. This is because in certain areas, such as in the vicinity of adiabatic surfaces, flowfield gradients may be very small, and the first rule is not stringent enough to adequately capture flowfied physics.

\section{Adaptation Methodology}

Intuitively, it appears that functions advantageons for grid adaptation should have properties pertinent to important aspects of the flowfield as well as having properties advantageous to fulfilling the rules cited above. Regarding the first criterion, areas where strong gradients occur are often characterized by a non negligible "gradient Knudsen number," $K n_{\alpha}{ }^{5}$ The reciprocal length scale $\left(l_{\alpha}\right)^{-1}$ of this Knudsen number is comprised of the local change in some flow variable $\alpha$ along its direction of strongest change $\zeta$, divided by the local value of the flow variable itself

$$
\left(l_{\alpha}\right)^{-1} \equiv\left|\frac{1}{\alpha} \frac{\partial \alpha}{\partial \zeta}\right|=\left|\frac{\partial \ln \alpha}{\partial \zeta}\right|
$$

(iradient length scales appear to be desirable quantities to use as physical length scales for grid adaptation. As just noted however, variables chosen for $\alpha$, such as density, velocity, or temperature, while inportant in much of the flowfield, nay not accurately reflect changes near certain surfaces. To satisfy the second criterion, we use $\lambda$ as another physical length scale for grid adaptation. Presently $\lambda$ is computed using the equilibrimm relationship. Although this formula is not valid where translational nonequilibrium exists, it estimates $\lambda$ well enough for initial adaptation purposes at this stage of the investigation.

Using reciprocals of these physical length scales, we construct functions to adapt initial grids from the solutions they generate. To date, the regions comprising the flowfield are adapted in one direction only, using either rows or columns of cells in the body-fitted mesh. In Fig. 1, these directions are denoted by $\xi$ and $\eta$, respectively. Ciradients are calculated using values contained in cells and their nearest neighbors in the direction of adaptation. 'This approximation creates inaccuracies in terms of obtaining true gradients 


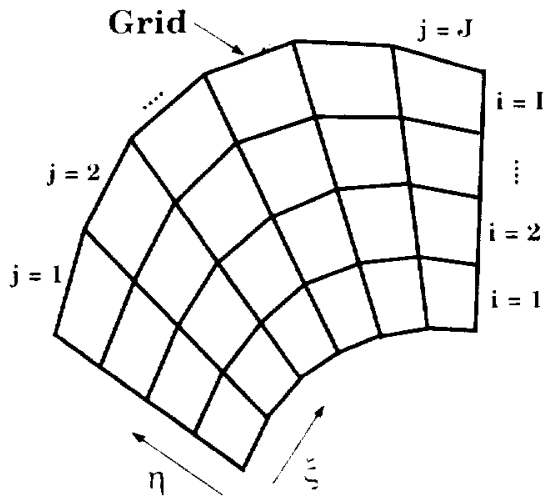

Fig. 1 Schematic representation of DSMC domain mesh.

with respect to a reference frame for the overall flowfield, but it provides a relative measure of them along the direction of adaptation. Although the following procedures are discussed in terms of column adaptation, the sarme applies for row adaptation as well.

An adaptation function $F$ is generated from the absolute reciprocal values of these lengths by computing their curmulative sum up through each cell in the direction of the grid adaptation. This function increases monotonically, and has its steepest slopes where the inverse lengths are greatest. Typical behavior of $F$ is shown in Fig. 2c. For colunn adaptation, $F$ is a function of $\eta$, and for a constant number of cells per column, cell spacing $\Delta \eta$ is altered through the adaptation process by inverting the function. In this manner, the new cell spacing $\Delta \eta^{\prime}$ is given by equal increments of $\hat{F}$, and $\Delta \eta^{\prime}$ will be minimized where the slope of $\dot{F}$ is steepest. A typical value in a term for altering $\Delta \eta$ within a column of cells in an $I \times J$ matrix is computed the following way

$$
\hat{F}\left(m, j ; l_{\alpha}\right)=\sum_{i=1}^{m} \frac{\Delta \eta(i, j)}{l_{\alpha}(i, j)} / \sum_{i=1}^{I} \frac{\Delta \eta(i, j)}{l_{\alpha}(i, j)}
$$

As the subscripts indicate, each column of cells has a separate function $\hat{F}$ for adaptation. If $\hat{F}$ is normalized by its final value, as in Eq. (2), so that values lie between zero to unity, a number of such functions, using different variables for $\alpha$, may be combined to create a composite function $\hat{C} i$. Additionally, the individual terms of this composite function may be weighted so that they dominate $G$ where they are strongest.

These ideas were tested for rarefied flow over the front half of a cylincler. Fig. 2a shows the initial grid and geometry used in the simulation, which took advantage of Howfield symmetry about the stagnation streamline. The freestream Mach, Reynolds, and Knudsen number values were $M_{\infty}=3.1$, Rer, $=88.6$, and $K n_{r, \infty}=0.052$, respectively, based on cylinder radius, and the cylinder's surface temperature was equal to the freestream stagna- 

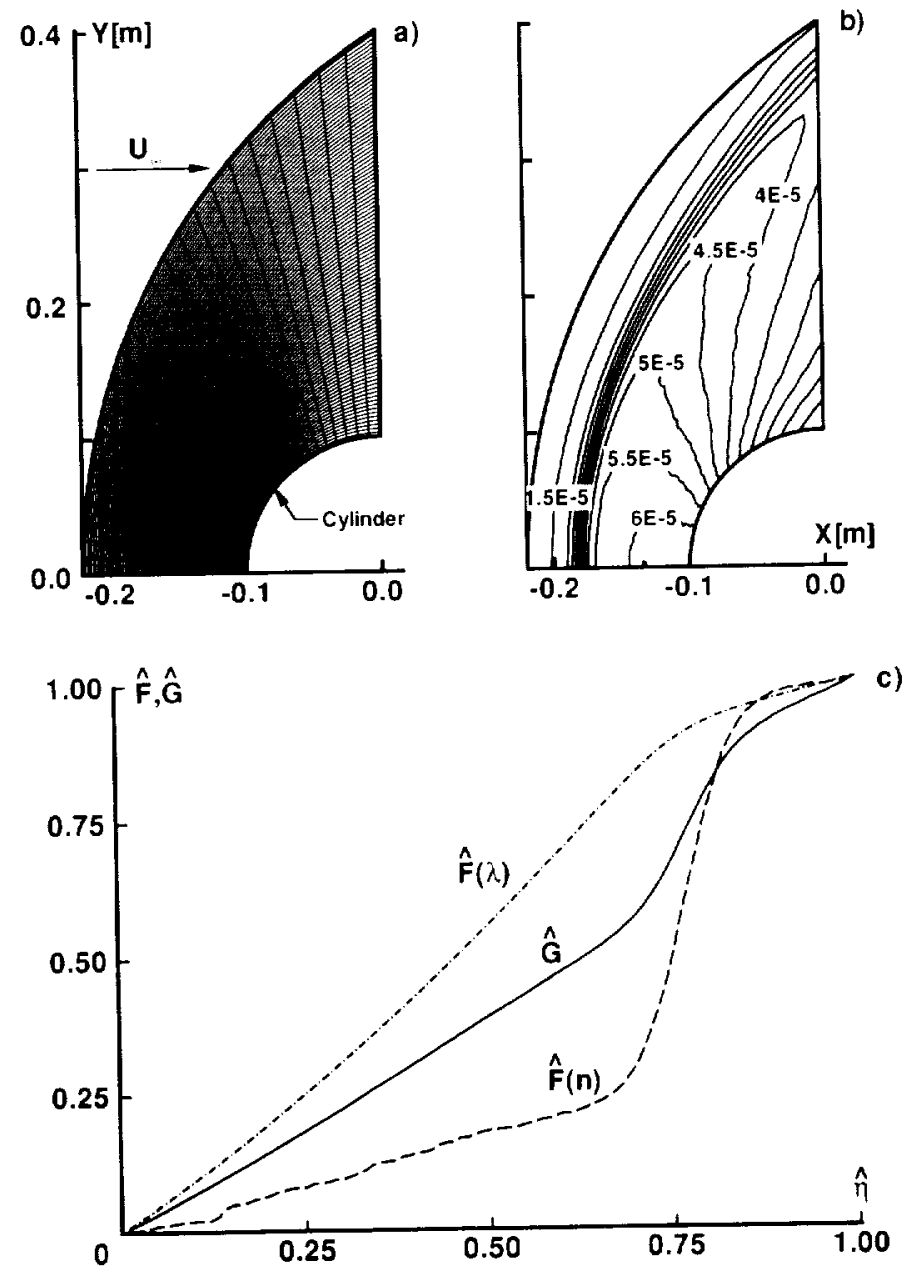

Fig. 2 Flow over cylinder, $M_{\infty}=3.1, R e_{r, \infty}=88.6, K n_{r, \infty}=0.052, \&$ $T_{w} / T_{\infty}=2.92$ : (a) original mesh, (b) density contours $\left[\mathrm{kg} / \mathrm{m}^{3}\right]$, (c) variation of $\hat{F} \& \hat{G}$ for cells nearest centerline.

tion temperature $\left(T_{\infty}=300 \mathrm{~K}, T_{w}=T_{0}=877 \mathrm{~K}\right)$. Density contours from the results are shown in Fig. $2 \mathrm{~b}$. A composite function $\hat{G}$ was developed to emphasize tight cell spacing near the surface and through the shock wave to adequately capture surface fluxes and flowfield gradients through the shock wave. The function adapted cell spacing $\Delta \eta$ nominally normal to the cylinder's surface, and was weighted towards $\lambda$ near the surface and towards $l_{n}$, the gradient length scale based on number density $n$, near the outer boundary. The specific form of $G$ became

$$
\begin{aligned}
\hat{G}(m, j)= & \left(\frac{\eta(m, j)}{\eta(I, j)}\right)^{\beta} \hat{F}\left(m, j ; l_{n}\right) \\
& +\left(1-\left(\frac{\eta(m, j)}{\eta(I, j)}\right)^{\beta}\right) \hat{F}(m, j ; \lambda)
\end{aligned}
$$



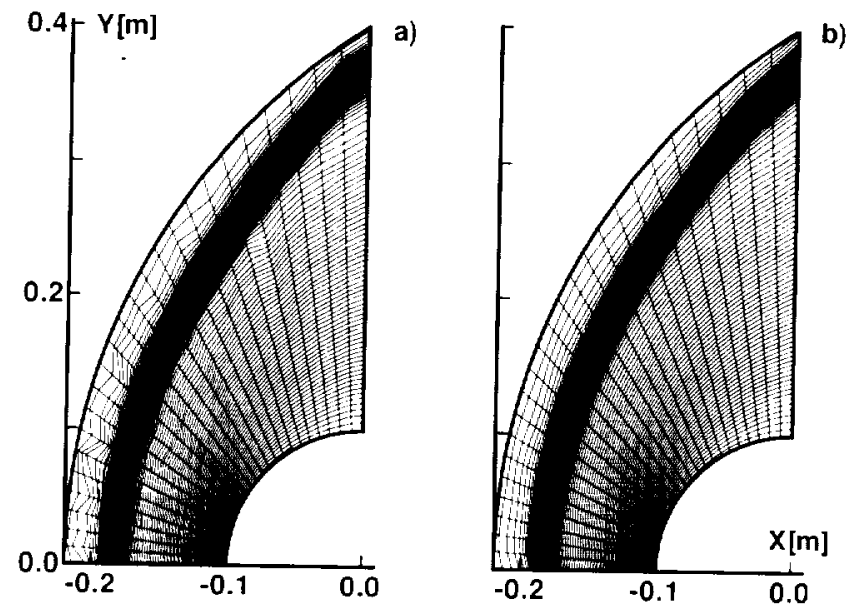

Fig. 3 Meshes adapted to solution depicted in Fig. 2: (a) adapted to Eq. (3) without filtering, (b) same, with slight FFT filtering.

The weighting factor was controlled by the free parameter $\beta$, an exponent whose value was set to unity for linear weighting. The variation of $G$ and its constituents with distance away from the cylinder near the stagnation streamline are shown in Fig. 2c.

Fig. 3a shows the adapted mesh resulting from application of Eq. (3) to the initial results. Comparing Fig. 3a and Fig. 2a, it is apparent that mesh lines are now strongly biased towards the location of the shock wave and near the cylinder's surface in the stagnation region.

\section{Handling of Statistical Fluctuations}

Fig. 3a also reveals a complication in the grid adaptation process. Note the "herringbone" pattern resulting from the statistical nature of particle methods, which is accentuated in regions of large $l_{n}$. Cell-averaged flowfield properties have a certain level of statistical scatter, or "noise" associated with them. In order to shorten the iteration process, we wish to adapt the mesh to solutions with small levels of sampling, which magnifies the effects of statistical scatter. This problem is further aggravated by adapting to flowfield gradients, obtained by differencing statistically noisy values.

Efforts to use least-square fits of polynomials and conic sections to the adapted grid lines proved ineffective. Such fits shifted tight grid spacing away from certain areas where it was physically needed. We also evaluated different filters to reduce the noise in the data set and/or the altered mesh. They were: centrally weighted averaging, median filtering, ${ }^{6}$ and fast fourier transform ( $\mathrm{FF}^{\prime}$ ) filtering. ${ }^{7}$ (Of these, FFT filtering has been the most effertive at reducing the high-frequency noise in our data sets. However, it can 
produce low-frequency variations in values of data sets or in grid spacing, sometimes shifting tight grid spacing away from where it is needed. Nevertheless, we retained it as a tool for producing acceptable grids. Fig. 3b shows the result of applying FFT filtering to the mesh depicted in Fig. 3 a.

The most successful method for reducing statistical scatter encountered thus far resulted from observations concerning $\hat{F}$ and $\hat{G}$. These functions adapt to data in only one direction, and for column adaptation each column of cells is adapted independently. There is no regard for the fact that final values the denominators receive before normalization vary across columns. In Fig. 4, the values of $\hat{F}$ denominators for each column of the simulation depicted in Fig. 2 are shown. The first column represents cells closest to the flowfield centerline, and the last column represents those nearest the exit plane. Such variation depends on mesh geometry, over which $F$ denominators may vary widely. When $\hat{F}$ or $\hat{G}$ is used where no strong gradients exist, the functions tend to adapt to the spiky noise itself. We preferred preserving features of the original grid where this occurs.

A variable coefficient $\Gamma\left(I, j ; l_{a}\right)$ was created for $\hat{F}$, equal to the ratio of the denominator of $\hat{F}$ for each column to the maximum value of $\hat{F}$ denominators across all columns. The unitary complement of this coefficient then multiplies $\sum \Delta \eta / \eta$, the incremental arc length of the original grid itself. The coefficient $\Gamma\left(I, j ; l_{\alpha}\right)$ and the modified function $\tilde{F}$ are given by

$$
\Gamma\left(I, j ; l_{\alpha}\right) \equiv \sum_{i=1}^{I} \frac{\Delta \eta(i, j)}{l_{\alpha}(i, j)} /\left(\sum_{i=1}^{I} \frac{\Delta \eta(i, j)}{l_{\alpha}(i, j)}\right)_{\max }
$$

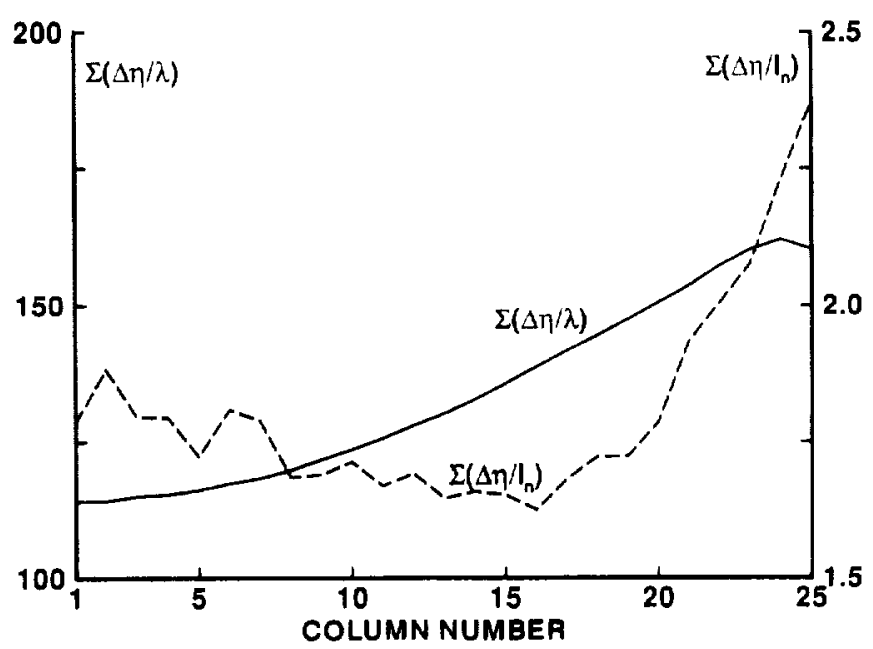

Fig. 4 Variation of $\hat{F}$ denominators across columns for solution depicted in Fig. 2. 
and

$$
\hat{F}\left(m, j ; l_{\alpha}\right) \equiv \Gamma\left(I, j ; l_{\alpha}\right) \hat{F}\left(m, j ; l_{\alpha}\right)+\left(1-\Gamma\left(I, j ; l_{\alpha}\right)\right) \sum_{i=1}^{m} \frac{\Delta \eta(i, j)}{\eta(I, j)}
$$

Since $F$ ranges from zero to one, the impact of individual $F$ variations on grid spacing are de-emphasized along columns where their summations are relatively small. Again, $\tilde{F}$ functions based on different variables may be combined to create composite functions, herein denoted by $\vec{i}$.

\section{Results}

In Fig. 5a, we show an initial grid consisting of four separate regions, once again for flow over a cylinder. In this example, the freestream conditions consisted of $M_{\infty}=6.0, R e_{r, \infty}=684, K n_{r, \infty}=0.013$, and $T_{\infty}$ $=63 \mathrm{~K}$. The surface temperature was $T_{w}=300 \mathrm{~K}$. Since the overall governing Knudsen number places this simulation in the near-continuum regime, multiple regions enhanced resolution in the vicinity of the bow shock and in the viscous region near the surface. Simulation results are qualitatively similar to those depicted in Fig. 2b. We fabricated the grid shown in Fig. 5b using the following composite function $\tilde{G}$, based on $l_{n}$ and $\lambda$

$$
\begin{aligned}
\tilde{G}(m, j)=( & \left.\frac{\eta(m, j)}{\eta(I, j)}\right)^{\beta} \Gamma\left(I, j ; l_{n}\right) \hat{F}\left(m, j ; l_{n}\right) \\
& +\left(1-\left(\frac{\eta(m, j)}{\eta(I, j)}\right)^{\beta}\right) \Gamma(I, j ; \lambda) \hat{F}(m, j ; \lambda) \\
& +\left(2-\Gamma\left(I, j ; l_{n}\right)-\Gamma(I, j ; \lambda)\right) \sum_{i=1}^{m} \frac{\Delta \eta(i, j)}{\eta(I, j)}
\end{aligned}
$$

By using $\lambda$ as one of the variables for adaptation, we expected to reduce in the number of cells violating the rule regarding grid spacing versus $\lambda$ in areas of enhanced resolution. Diagnostics in the adaptation code confirm this feature for this case. In the initial solution, the fraction of cells violating this rule was $0.1 \%$ in Region 1 (near the surface), and $24 \%$ in Region 3 (through the shock wave). It should be noted that few of these cells grossly violated the rule, however. After repeating the simulation on an adapted grid without FFT smoothing for approximately the same number of steadystate timesteps, this rule was violated only $0 \%$ and $9 \%$ in those respective regions.

Up to this point, the simulations presented here have arguably had adequate resolution before undergoing grid adaptation. Our final example demonstrates that this adaptation process can increase solution accuracy for a given grid with a fixed number of cells and regions even when the initial solution suffer; from inadequate resolution. This case stems from a series of simulations originally run in conjunction with a DSMC (investigation of a 

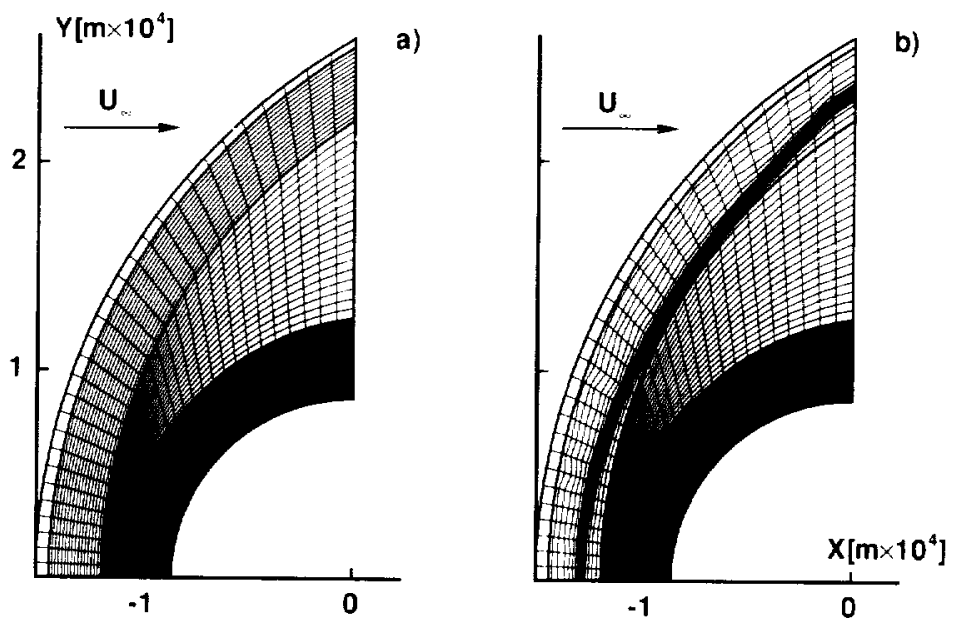

Fig. 5 Multiple-region mesh for calculating flow over cylinder, $M_{\infty}$ $=6.0, R e_{r, \infty}=684, K n_{r, \infty}=0.013, \& T_{w} / T_{\infty}=4.76$. Only every fifth "tangential" grid line shown for clarity: (a) original mesh, (b) mesh adapted to initial solution using Eq. (6), without filtering.

shock-shock interaction problem. ${ }^{1}$ Data from the interaction problem was used to model the shear layer that develops beyond the point where the shock waves intersect. The simulation was run for three different levels of resolution approximately normal to the flow direction. The medium resolution grid has four times as many cells in the $y$-direction as the coarsest mesh, and the highest resolution grid has sixteen times as many cells in that direction as the coarsest mesh. It is considered that only the final mesh had adequate resolution. ${ }^{1}$ Fig. 6 depicts velocity magnitude contours from these high-resolution results.

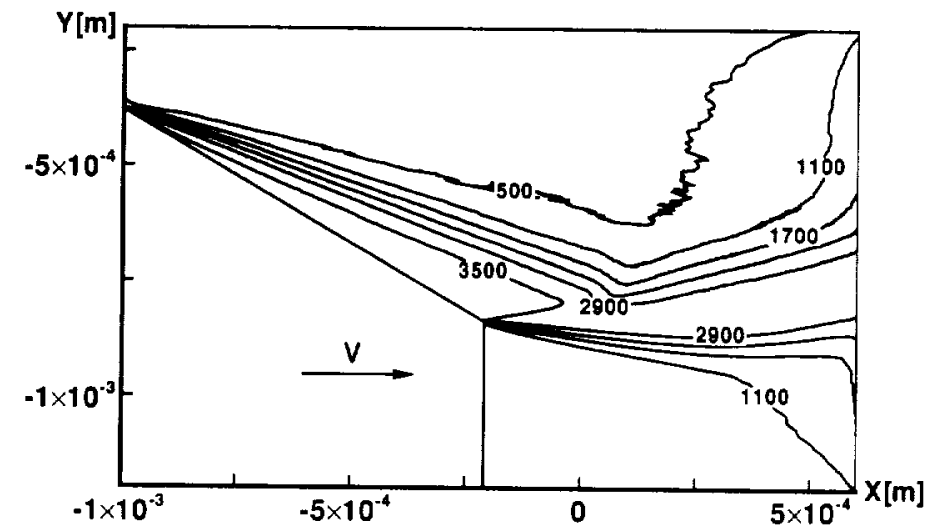

Fig. 6 Shear flow generated using conditions from separate shock-shock interaction problem. Velocity magnitude contours $[\mathrm{m} / \mathrm{sec}]$. 

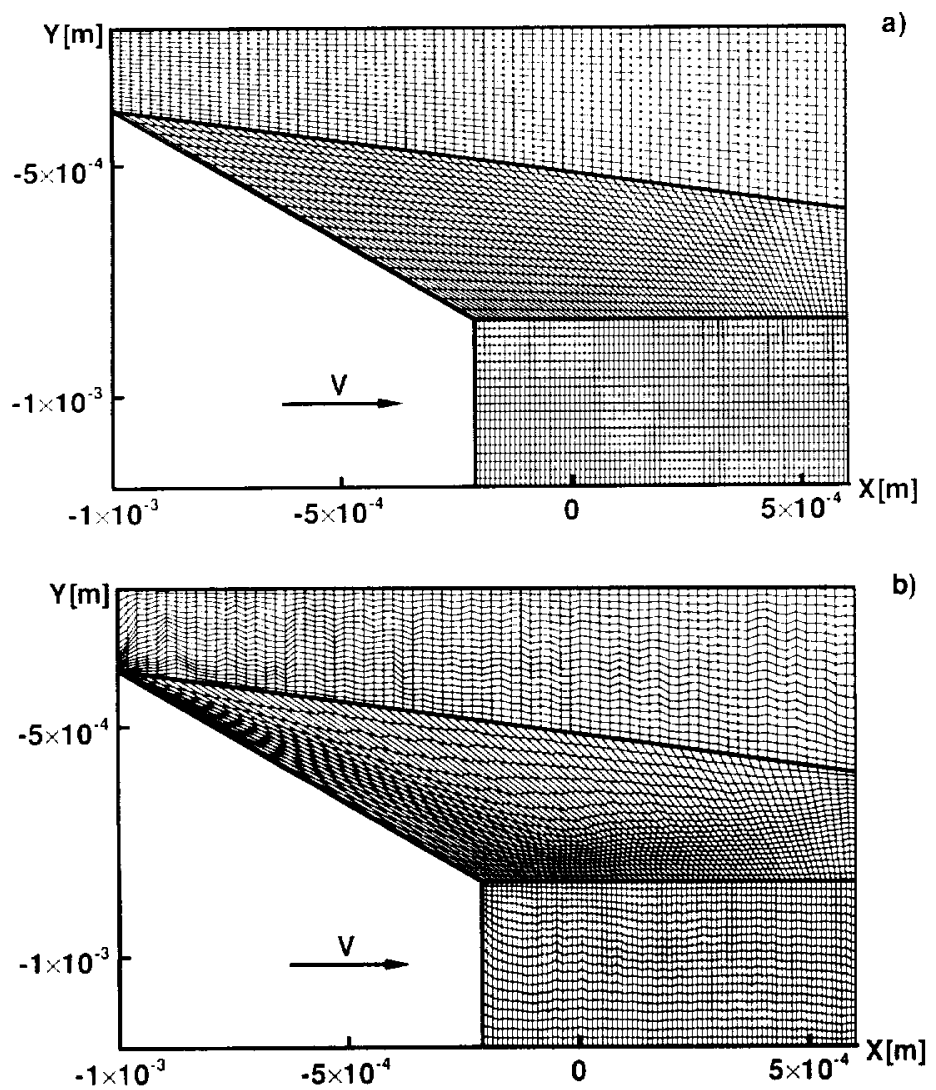

Fig. 7 Meshes used to calculate flowfleld depicted in Fig. 6: (a) original mesh, (b) mesh adapted to initial solution using modifled version of Eq. (6) with slight FFT filtering.

In Fig. $7 \mathrm{a}$, we show the initial grid used in the coarsest simulation. Investigating the effects of different length scales on adaptation in this particular case led us to the grid depicted in Fig. 7b. This was generated using a composite function $\underset{G}{f}$ similar to that described in Equation (6), using length scales based on $l_{n}$ and $\lambda$. The only difference was to replace the factor

$$
\left(\frac{\eta(m, j)}{\eta(I, j)}\right)^{\mathcal{\beta}}
$$

with $1 / 2$ because this flowfield did not require the type of weighting desired for the bounded flowfields depicted earlier. The length scale based on $\lambda$ was included to dampen noisy contributions from $l_{n}$.

One run was generated using the grid adapted to the coarsest mesh results. Another run was generated using a grid adapted to the medium 


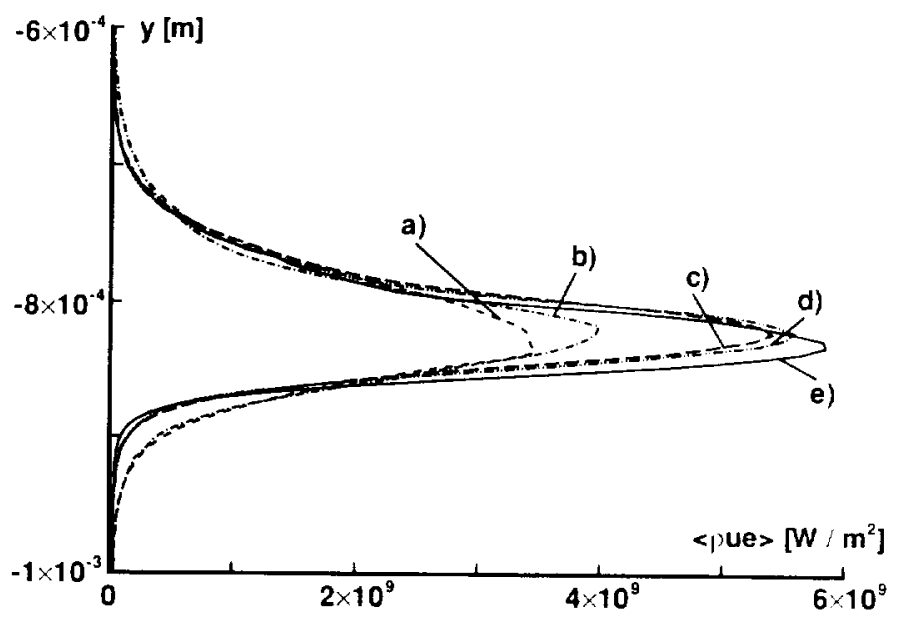

Fig. 8 Profiles of $<\rho u e>$ for different meshes used to calculate flowfleld of Fig. 6 at $x=0$ : (a) unadapted/coarse, (b) adapted/coarse, (c) unadapted/medium-resolution, (d) adapted/medium-resolution, and (e) unadapted/high-resolution.

resolution mesh using the same function $\tilde{G}$. (Contour plots of the adapted grid results appeared virtually identical to their respective original cases. However, as depicted in Fig. 8, profiles of the energy flux <pue $>$ at a plane across $x=0$, a location containing some of the steepest gradients, demonstrate the relative effectiveness of the grid adaptation technique. The peak flux from the solution on the coarse adapted grid is $15 \%$ higher than that from the plain coarse grid, and the solution from the medium-level adapted grid shows a peak energy flux $4 \%$ higher than its progenitor. Also, the gradients captured by the adapted grids are slightly steeper than their respective original cases, and occur over a narrower band. The full-width/halfmaximum value of $\langle\rho u e\rangle$ for the coarse adapted grid is $86 \%$ of the original coarse-grid solution, and that for the medium adapted grid is $96 \%$ of the unadapted medium-resolution result. In both peak flux and flux width, the trend of data is toward the high-resolution result when going from original to adapted-grid solutions.

\section{Considerations for Future Work}

Although we have addressed the rules mentioned in the Discussion section, we have made no provision guaranteeing an adequate number of particles per cell for proper collision mechanics. Additionally, for optimum use of computational resources, we desire roughly equal numbers of particles per cell. This rule conflicts with cell resolution guidelines, since the local cell population is proportional to the product of local density and cell volurne. The conflict can be resolved somewhat by using variable particle 
scale factors, a concept already extended by olynick to the limit where rach cell is troated as a separate region. "It may be desirable to combine current adaptive mesh procedures with such an approach.

'The last example illustrates that in adapting to an initial solution with grossly inadequate resolution, the level of improvement is constrained by fixing the mumber of cells and regions to those of the initial solution. It would be useful to automatically increase or decrease cell resolution by changing the number of cell rows or columns allotited to a given region based on the initial solution. It may even be advantageous to relax the constraints on the locations of certain outer boundaries (ahead of a bow shock into the freestean, for example).

It would also be advantageous to adapt rows and columns of cells simultaneously for enhanced ability to capture governing flowfield phenomena, within the constraints imposed by proper collision mechanies. Finally, restarting calculations from existing sohutions on adajted grids, rather than beginning new computations from scratch, would greatly reduce the amomnt of time spent achieving new steady-state solutions.

\section{Conclusions}

Wo have begun developing a technique for adapting DSMC: meshes to flowfield solutions. The functions used for adapting these grids are based on length scales that are physically meaningful in terms of satisfying certain empirical rules usually fulfilled manually. The formulation of these adaptation functions is flexible enough that they may be used for unbounded Howfields as well as flows past bodies. Use of this technique can improve resolution without requiring greater resources in terms of numbers of cells and particles, or the extra CPU time and memory necessary to solve a larger simulation.

\section{Acknowledgments}

This work was supported by NASA Contract No. NAS1-19237. We appreciate David Myrick's helpful suggestions regarding filtering.

\section{References}

'Garlson, A. B., and Wilmoth, R. (1. "Monte Carlo Simulation of a NearContinum Shock-Shock Interaction Problem," AIAA Paper No. 92-2862, AIAA $30{ }^{t h}$ Aerospace Sciences Meeting, Reno, Nevada, January 1992.

${ }^{2}$ Dogra, V. K., Moss, J. N., Wilmoth, R. C., and Price, J. M., "Hypersonic Rarefied Flow Past Gpheres Including Wake Structure," AIAA Paper No. 920495, AlAA $30^{t h}$ Aerospace Sciences Meeting, Reno, Nevada, January 1992.

${ }^{3}$ Rault, [). F. (A, "Aerodynamic Characteristics of a Hypersonic Viscous (Optimized Waverider at Iligh Altitudes," AIAA Paper No. 92-03306, AIAA 30 ${ }^{\text {th }}$ Aerospace Sciences Meeting, Reno, Nevada, January 1992.

${ }^{4}$ Woronowicz, M.S., "Application of a Vectorized Particle Simulation to the Study of Plates and Wedges in High-speed Rarefied Flow" Ph. D. Thesis, Department of Atronaltics and Astronautics, Stanford University, Stanford, (A, I Ine 1991. 
${ }^{5}$ Bird, (i. A., "Direct Simulation of (ias Flows at the Molecular Level," Communications in Applied Numerical Methods, Vol. 4, No. 2, March-April, 1988, pp. $165-172$.

"Justusson, B. I., "Median Filtering: Statistical Properties," Two-Dimensional Digital Signal Processing II, edited by T. S. Huang, Springer-Verlag, New York, 1981, pp. 161-196.

${ }^{7}$ Press, W. H., Flannery, B. P., Teukolsky, S. A., and Vetterling, W. T., "Numerical Recipes: The Art of Scientific Computing," Cambridge Oniv. P'ress, New York, 1985, Chapter 12.

${ }^{8}$ Olynick, D. P. Moss, J. N., and Hassan, H. A., "Grid Generation and Adaptation for the Direct Simulation Monte Carlo Method," AIAA Paper No. 88-2734, AIAA $23^{\text {rd }}$ Thermophysics Conference, Sall Antonio, Texas. 

